\title{
Interprofessional Healthcare Student Perceptions of Clinical vs. Simulation Learning Through Participation in Underserved Health Clinics
}

\author{
Amy Christopher ${ }^{1}\left[\right.$ ] $\cdot$ Tina Gortemiller ${ }^{2} \cdot$ Jonathan Zemmer $^{3} \cdot$ Meredith Wronowski $^{4}$ (I)
}

Accepted: 23 April 2021 / Published online: 11 May 2021

(C) International Association of Medical Science Educators 2021

\begin{abstract}
Purpose The purpose of this research project is to determine how well three health profession student discipline's learning needs are met through a real clinical interprofessional environment compared to previous simulation experience by assessing student perceptions following participation in a student-run underserved healthcare setting.

Methods Various student-run health clinics were initiated throughout Southwest Ohio in underserved settings precepted by healthcare profession faculty. Physician assistant, pharmacy, and medical student learning needs and interprofessional perceptions were assessed following clinic participation utilizing the Clinical Learning Environment Comparison Survey (CLECS) and qualitative comment to allow objectification of students' perceptions to different clinical learning environments and the interprofessional setting.

Results The survey had responses from medical, pharmacy, and physician assistant students $(n=60)$. Overall, students preferred traditional learning environments compared to previous simulated clinical experiences $(p<.01)$. Significance for communication, holism, and the teaching-learning dyad was demonstrated comparing the traditional clinical experience to the simulated experience $(p<.05)$. Pharmacy students felt their critical thinking skills were more significantly improved after the simulated environment $(p<.05)$. Males more significantly favored teaching-learning dyad simulation vs. the traditional environment $(F=5.86, p<.05)$. Qualitative comments regarding the interprofessional experience included themes of teamwork, patient communication, and clinical skills.

Conclusion When comparing a traditional clinical experience in an interprofessional student-run clinic vs. previous simulated learning clinical environment, physician assistant, pharmacy, and medical students qualitatively and quantitatively perceive their learning needs are greater met in the volunteer student-run clinic for communication, holism, and the teaching-learning dyad. There were variations in preferences based on sex and discipline of the student.
\end{abstract}

Keywords Clinical education · Simulation · Experiential learning · Interprofessional education · Student-run clinics · Underserved

\section{Introduction}

Throughout various disciplines of healthcare, including medicine, nursing, pharmacy, dentistry, and physician assistants, students must learn how to interact with patients while

Amy Christopher

achristopher1@udayton.edu

1 University of Dayton, Fitz Hall, 300 College Park, Dayton, $\mathrm{OH}$, USA

2 University of Cincinnati, Cincinnati, OH, USA

3 Wright State University, Dayton, OH, USA

4 University of Dayton, Dayton, OH, USA also being able to form a clinical plan efficiently [1]. There are two ways this can be accomplished. The first option is interacting with a patient in a simulated learning environment. Simulations connect classroom learning with future occupational and concrete clinical experience. A simulation can be in a high-fidelity experience or with a patient actor. This can be accomplished with an OSCE (Objective Structured Clinical Examination) with a hired actor, the use of computerized manikins, or another healthcare professional student who has been given a script to learn, with predefined answers to questions [2]. Some didactic curriculum simulations are executed in a small group or team setting and may include an interprofessional component. The second option is learning in a real-life clinical setting, such as a 
student-run free clinic or student clinical experience. In this setting, the student is interacting with actual patients. There are no scripts involved and all decisions made in this setting will be applied to a real patient.

The purpose of this research project is to contrast the student's learning and interprofessional experience in previous simulated clinical experience to a real clinical interprofessional environment by assessing student perceptions immediately after participation in an interprofessional underserved healthcare setting. This pilot study utilizes a clinical learning comparison survey and qualitative questions to assess the student's learning experience in the two environments.

The underserved student-run free clinic setting is interprofessional due to the intentional student pairing of the three different health profession disciplines by precepting faculty while they collaborate to address patient's medical and medication needs. According to the Society of StudentRun Free Clinics [3], free clinics are staffed by student volunteers that seek opportunities to provide care to the uninsured, underinsured, or homeless while enriching education with real-life patient care. These clinics identify social service opportunities for populations with limited access to clinical care. A student-run clinic in the Boston area identifies the aim of student-run clinics as broadening students' understanding of the healthcare system by engaging them in service-oriented activities, health care management, quality improvement, research, and patient education [4]. A study in 2007 described the role of a student-run free clinic as students take responsibility for management of a healthcare clinic while also being able to prescribe medication for specific and common disease states [5]. Other student-run free health clinics have involved physician assistant, pharmacy, and medical students, but the focus of outcomes in a recent free clinic involving these professions was on IPE student perceptions [6-9] without investigating student learning needs being met by clinical compared to simulation educational experiences. Student perceptions of the role of various health professions were investigated in a smoking cessation-only free student clinic involving these three professions [7]. Similarly, this study did not investigate the learning needs compared to didactic curriculum exposure to patient care. The most recent study involving these 3 disciplines in student-run medication review clinic focused on outcomes of clinical therapeutic interventions with a minor focus on the interprofessional experience [8]. There remains debate on which method of learning is more effective and thus the importance of this research is proven $[10,11]$. The importance of this pilot study is to help answer whether student perceptions of learning needs being met in the two educational settings when comparing the traditional clinical experience in an IPE student-run clinic.
Student perceptions of learning needs being met by clinical education in an interprofessional, student-run free clinic compared to simulation education were assessed by utilizing the Clinical Learning Environment Comparison Survey (CLECS) [12], which was validated by 11 nursing and one research expert. The survey allows objectification of students' perceptions of how learning needs were met by different clinical learning environments. CLECS utilizes a Likert scale evaluating six different subscales of self-efficacy, the teaching-learning dyad, holism, communication, the nursing process, and critical thinking. CLECS can be used to evaluate the facilitator and evaluate the simulation versus clinical experience [12]. The CLECS also assesses two questions regarding communication with and support of the interprofessional team (see Table 1).

The interprofessional education collaborative (IPEC) [13] competencies of teams and teamwork, roles and responsibilities, and interprofessional communication apply to the student-run clinics by including medical students, physician assistant students, and pharmacy students. These competencies are accomplished through the collaborative care during the clinics. Medical, physician assistant, and pharmacy students collaboratively apply their health profession training knowledge during patient history taking and work together to improve health outcomes for underserved patients. Each student member of the team could apply their developing expertise. Medical and physician assistant students both assess the patient and identify a medical plan of action for each patient. The pharmacy students take medication history, counsel on new and old medications, and aid the medical and physician assistant students in initiating the most effective medication plan for patients with their pharmaceutical knowledge. Communication is key in this setting for not only ensuring that the patient understands what is happening with their health, but also to make sure all members of the health care team agree with and thoroughly understand the treatment plan for the patient.

A similar survey in 2018 was utilized by faculty of a student-run clinic in the medical, nursing, and physiotherapy fields [14]. This mixed methods study out of Sweden used the Clinical Learning Environment, Supervision, and Nurse Teacher Evaluation Scale (CLES + T). During this study, surveys were completed by students following a student-run clinic to assess satisfaction with the learning environment. Patients were also surveyed regarding client satisfaction questionnaire surveys (CSQ-8) focusing on the care they received while at the clinic, and then, semi-structured interviews were conducted with the supervisors and students after the clinic ended. The study found patient and student appreciation of the pedagogical and patient-centered care of the primary health care clinic [14].

To date, there are few studies that have investigated health care student's perceptions of traditional clinical vs. 
Table 1 Descriptive statistics and reliability of clinical learning environment comparison survey

\begin{tabular}{|c|c|c|c|c|c|}
\hline Item & $N$ & Min & $\operatorname{Max}$ & Mean & SD \\
\hline \multicolumn{6}{|l|}{ Communication scale $(\alpha=0.81, N=55)$} \\
\hline Preparing to care for patient & 113 & 2 & 4 & 3.47 & 0.63 \\
\hline Communicating with interdisciplinary team & 104 & 1 & 4 & 3.14 & 1.03 \\
\hline Interacting with patient & 115 & 1 & 4 & 3.56 & 0.70 \\
\hline Providing information and support to patient's family & 60 & 1 & 4 & 2.67 & 1.11 \\
\hline \multicolumn{6}{|l|}{ Healthcare process $(\alpha=0.87, N=106)$} \\
\hline Understanding rationale for patient's treatment plan & 116 & 1 & 4 & 3.36 & 0.75 \\
\hline Understanding patient's pathophysiology & 112 & 1 & 4 & 3.20 & 0.80 \\
\hline Identifying patient's problems & 116 & 2 & 4 & 3.57 & 0.58 \\
\hline Implementing care plan & 110 & 1 & 4 & 3.42 & 0.73 \\
\hline Prioritizing care & 115 & 1 & 4 & 3.40 & 0.77 \\
\hline Performing appropriate assessment & 114 & 1 & 4 & 3.53 & 0.68 \\
\hline \multicolumn{6}{|l|}{ Holism $(\alpha=0.89, N=66)$} \\
\hline Assessing outcomes of the care provided & 97 & 1 & 4 & 3.06 & 0.98 \\
\hline Identifying short- and long-term healthcare goals & 95 & 1 & 4 & 3.06 & 0.99 \\
\hline Discussing patient's psychosocial needs & 105 & 1 & 4 & 2.96 & 1.02 \\
\hline Discussing patient's developmental needs & 94 & 1 & 4 & 2.90 & 1.05 \\
\hline Discussing patient's spiritual needs & 83 & 1 & 4 & 2.43 & 1.08 \\
\hline Discussing patient's cultural needs & 88 & 1 & 4 & 2.61 & 1.12 \\
\hline \multicolumn{6}{|l|}{ Critical thinking ( $\alpha=0.91, N=87$ ) } \\
\hline Anticipating and recognizing changes in patient's condition & 97 & 1 & 4 & 3.09 & 0.94 \\
\hline Taking appropriate action when patient's condition changes & 91 & 1 & 4 & 3.12 & 0.93 \\
\hline \multicolumn{6}{|l|}{ Self-efficacy $(\alpha=0.76, N=87)$} \\
\hline Reacting calmly to changes in my patient's condition & 92 & 1 & 4 & 3.21 & 0.96 \\
\hline Knowing what to do if I make an error in my care & 99 & 1 & 4 & 2.86 & 0.99 \\
\hline Being confident in my decisions & 111 & 1 & 4 & 3.33 & 0.75 \\
\hline Feeling confident in my healthcare abilities & 116 & 2 & 4 & 3.37 & 0.72 \\
\hline \multicolumn{6}{|l|}{ Teaching-learning dyad $(\alpha=0.76, N=111)$} \\
\hline Having my instructor available to me & 116 & 1 & 4 & 3.59 & 0.75 \\
\hline Feeling challenged and stimulated & 115 & 1 & 4 & 3.57 & 0.62 \\
\hline Receiving immediate feedback on performance & 111 & 1 & 4 & 3.28 & 0.90 \\
\hline $\begin{array}{l}\text { Feeling supported by instructor and peers when making care } \\
\text { related decisions }\end{array}$ & 114 & 1 & 4 & 3.50 & 0.72 \\
\hline Improving my critical thinking skills with experience & 114 & 2 & 4 & 3.65 & 0.59 \\
\hline
\end{tabular}

simulation experience especially in an underserved clinic setting. Physician assistant student perceptions of experiential education in an underserved student-run free clinic have not been well studied [11]. From an international perspective, advanced prescribing practitioner's clinical, experiential education, and interprofessional student perceptions are sparse even though the increase in prescribing by non-physician practitioners have increased over the past two decades [15]. Nursing and other advanced prescribing practitioner numbers have risen in Europe, Africa, Australia, and Canada during the twenty-first century [15]. The interprofessional aspect of a student-run clinic in an underserved clinic study is also of particular interest due to the current and recent pandemic's effects on clinical rotation availability [16].

The hypothesis of this research is that an interprofessional, student-run clinic would better meet the learning needs of medical, pharmacy, and physician assistant students compared to simulation education experiences as measured by student responses to the CLECS and qualitative comments. Comparing the student learning perception survey results of these three professions in a variety of underserved, interdisciplinary health clinics provides a unique perspective compared to previous studies of student-run clinics. 


\section{Methods}

\section{Study Participants}

This pilot study involved a total of 60 students volunteering in various healthcare clinics for the underserved in two urban areas in southwestern Ohio. Students were recruited via email by their respective university program community service coordinators for volunteering. Thirty-two of the students participating were physician assistant students, 11 were medical students, and 17 were pharmacy students. There was one survey collected where the participant did not distinguish which discipline they were.

\section{Baseline Characteristics}

Students were compared by age, healthcare discipline, sex, previous IPE experience, university attended, and whether the student was currently in the didactic or clinical phase of their education. Two physician assistant students had not experienced any health simulations in their program, so the CLECS data was not collected. Physician assistant didactic students were in their first 16 months of didactic curriculum for a 27-month masters program. Pharmacy and medical students were in their second and third didactic year of a 3-4-year doctoral program. Study participants were not compensated nor had any obligation through coursework for a grade for clinic volunteering (see Table 2).

\section{Learning Environment Comparison: IPE Student-Run Underserved Clinic vs. Previous Simulation Experience}

\section{Student-Run Clinics, Clinic Structure, and Interprofessional Component}

Free health clinics were organized by various health professional educators in southwest Ohio. Clinic education materials were emailed to students prior to the clinic date. Physician assistant, pharmacy, and/or medical students were intentionally paired by precepting faculty with at least one other health care discipline student for each clinic to ensure an interprofessional experience. Clinic assessments of patients included blood pressure checks, pulse oximeter measurement, weight measurement, neck circumference (insomnia clinic only), medical chart intake, past and family medical history (including social and lifestyle history), and patient medication history. Medical students and physician assistant students both assessed blood pressure and pulse oximeter readings as well as potential unmet medical needs. The pharmacy students obtained medication history, counseled on new and old medications, and aided the medical and physician assistant students in initiating the most effective medication plan for patients with their pharmaceutical knowledge. The patient assessments helped identify an appropriate health plan of action presented to the faculty clinic preceptor for each patient. Once a care plan was approved by the preceptor, the students reviewed a patient action plan to the patient or shelter resident. Some students were also able to follow up with residents and their respective prescribers regarding smoking cessation, nicotine replacement product needs, and other identified medication issues or free health clinic referrals.

Student-run clinic types included smoking cessation clinic, insomnia clinic, breathe better clinic, and medication/medical history reviews. Clinics were held in facilities providing shelter or financial assistance for low-income community residents.

The smoking cessation clinic involved assessing patients' smoking habits and their readiness to change, explaining the impact smoking can have on their general and oral health, and giving the patients information and/or providing nicotine replacement products. The insomnia clinic involved collecting information regarding insomnia history, sleep quality, neck circumference to assess their risk of obstructive sleep apnea, sleep hygiene counseling, assessing patients' readiness to alter sleep habits, and assisted in modifying sleep habits. At the breathe better clinic, patients were counseled on identifying and avoiding various triggers for chronic lung disease, had their inhaler techniques assessed, and were counseled on the purpose/use/side effects of their medications. The medication or medical history review clinics were not disease specific. In addition to medication review, chief
Table 2 Baseline characteristics of study participants

\begin{tabular}{lllll}
\hline Student discipline & $\begin{array}{l}\text { Age in years } \\
(\text { median})\end{array}$ & Sex (\% female) & $\begin{array}{l}\text { Previous IPE experi- } \\
\text { ence }(\% \text { stated yes) }\end{array}$ & $\begin{array}{l}\text { In clinical phase of } \\
\text { curriculum }(\%) * * *\end{array}$ \\
\hline Physician assistant $(n=32)$ & 23.7 & $75 \%$ female & $94 \%(n=2)$ & $9 \%(n=3)$ \\
Pharmacy $(n=17)$ & 23.6 & $65 \%$ female & $88 \%(n=2)$ & $35 \%(n=6)$ \\
Medicine $(n=11)$ & 24.8 & $55 \%$ female & $91 \%(n=1)$ & $9 \%(n=1)$ \\
\hline
\end{tabular}

A significantly higher number of pharmacy students were in the clinical phase of the curriculum at the time of study compared to physician assistant or medical students. There were no other significant demographic or educational differences between students when comparing the three disciplines

$* * * \chi^{2}=17.60 ; \mathrm{p}<.001$ 
complaints made by the patient were addressed by the medical team and action plans included counseling and prescriptions from a charitable pharmacy.

Interprofessional communication also occurs among the students during the clinic as well as with the preceptor. Communication is key in this setting for not only ensuring that the patient understands what is happening with their health, but also to make sure all members of the health care team agree with and thoroughly understand the treatment plan for the patient. Students also could create patient charts, write progress notes, and make medication management recommendations to collaborating health care providers after the clinic is finished.

\section{Previous Simulation Clinical Experiences}

Simulation experience varied for physician assistant, pharmacy, and medical healthcare profession training programs. The method of simulation was similar among the professions, but the frequency, evaluation, and feedback did differ. For each profession's simulation experience, a clinical scenario was given to an individual student or team of students to navigate an assessment of clinical problems and plan to resolve identified medical issues.

Physician assistant students in this study are required to complete four or more OSCEs/simulations each semester of their program. These advanced prescribing practitioners are required to watch their own recorded simulation once during the didactic curriculum and submit a written self-reflection for grading purposes. Self-reflections in this setting are only required during their first professional year, but physician assistant students are given the opportunity to view recordings of simulation experiences throughout the curriculum if they desire.

Sixty-six percent of the pharmacy students involved in this study are required in their didactic program to complete one OSCE per semester, or two per academic year during the curriculum. This majority of pharmacy students are required to watch their own recorded simulation and submit a written self-reflection for grading purposes. Regarding 34\% of the pharmacy students involved in this study, their didactic program grades simulated learning experiences using various rubrics and standards from accrediting bodies such as the Accreditation Council for Pharmacy Education (ACPE). Students are assessed in an OSCE format at the end of each of their three didactic professional years.

Regarding medical student simulation experiences, two types of simulated experiences are utilized. The first is a simulated patient encounter (SPE) and the second is an OSCE. Students in this program perform one SPE and two OSCEs in their first year and three SPEs during their second year. During their third year, they begin their clerkships in addition to multiple OSCEs throughout the year. The last medical school didactic year consists of one OSCE. None of the medical students participating were in the fourth year, but one medical student was in their third year which contained an introduction to clerkships. Similar to physician assistant students, medical students also document their simulations in SOAP note format or postencounter questions. Unlike pharmacy and physician assistant students, medical students are not required to view and reflect upon their recorded simulation experiences; however, they are given the opportunity to do so if they desire. Self-reflections apart from viewing a recording of an OSCE in this setting are only required during their first medical school professional year.

\section{CLECS Utilization and Validity}

The CLECS survey was administered to students immediately after completing the student-run clinic patient encounter in order to assess student perceptions of the different learning environments. Students were instructed to compare the current experience of the student-run underserved clinical environment to the previous simulated encounters received during didactic education. Due to the original design of the survey for undergraduate nursing students, student volunteers were instructed to answer the question regarding "nursing process" as the "medical process". Students were able to choose "not applicable" as an answer to a question if they felt appropriate. Afterwards, the completed anonymous surveys were collected. Permission was obtained from the authors of the CLECS for research purposes, reproduction, and distribution to students.

Two of the CLECS questions quantitatively assess student's interprofessional attitudes regarding communication and feeling supported by their peers when providing care (see Table 1). Student interprofessional perceptions were also collected qualitatively regarding the student-run clinic interprofessional and clinical preparedness experience.

The content validity of the CLECS survey for physician assistants, medical students, and pharmacy students was verified for clinical appropriateness through survey of various discipline education experts. The CLECS validity for physician assistant students was agreed upon by seven physician assistant educators with cumulative experience of over 38 years of education in this field. Pharmacy student content validity was also verified by eight pharmacy educators with over 55 years of clinical pharmacy education. Applicability of the content of the CLECS survey for medical student education was validated by eleven medical prescribers with 112 years of education in this discipline. One of the content reviewers included one medical school faculty with 45 years of medical education experience. 


\section{Institutional Review Board and Student Participation Consent}

This research was approved by the Institutional Review Board at the University of Dayton. Wright State University and University of Cincinnati IRB stated it was not necessary to obtain IRB approval for deidentified student survey involvement. Informed consent was obtained from participants by voluntary participation after being presented with a written IRB form describing the survey research. Students could decline participation of any or all the survey research activities. The community clinics were strictly voluntary and declining participation did not have academic consequence.

\section{Quantitative and Qualitative Analysis}

Baseline characteristics were compared with independent variables including the type of clinical experience in separate models. The main effects and interaction of these variables with clinical experience were assessed. Post hoc univariate ANOVAs were examined for differences in specific scales for baseline characteristics that were near significance.

The two-section, 29-question CLECS and qualitative responses were recorded in a Microsoft Excel ${ }^{\circledR}$ sheet also containing student type and clinic type. Exploratory factor analysis was used to assess the statistical validity of the CLECS scales, and the reliability of the final scales was assessed by determining Cronbach's alpha using the SPSS scale function (see Table 1 for items and their descriptive statistics) [16]. The reliability of each of the scales, communication, nursing process, holism, critical thinking, selfefficacy, and teaching-learning dyad was acceptable, with alpha values ranging from 0.76 to 0.91 . Composite variables of each scale were created by taking the mean of the items for each scale. A Pearson bivariate correlation was conducted to assess the direction and strength of the relationship between CLECS scales (see Table 3). The primary intervention variable of interest in student clinical experience type, specifically, the student-run traditional clinical experience compared to a previous simulation. Multivariate analysis of variance (MANOVA) was used to assess the differences in CLECS scales between these two types of clinical experience $[17,18]$. Student sex, program type (physician assistant, pharmacy, or medical), university enrollment (University of Dayton, Wright State University, Cedarville University, and University of Cincinnati), previous interprofessional education (IPE) experience, and clinical education status were included with the clinical education type variable. Included in the MANOVA models were the main effects of these variables as well as the interaction of these variables with the clinical education type variable. However, due to the relatively small sample size, each variable was tested with the clinical education type variable in separate MANOVA models (see Fig. 1 for a visual representation of the analytical approach).

The open-ended response items included with the student survey were utilized as a source of qualitative data to examine the experiences of student clinicians. Content analysis was utilized beginning with an in vivo coding process, followed by identifying themes and categories that emerged from comparing responses across student clinicians [19, 20]. Two members of the research team each coded all openended survey responses. These two researchers compared initial codes and themes and categories to ensure there was a satisfactory level of agreement on the themes and categories.

\section{Results}

The overall CLECS student survey response rate was $100 \%$ as all students approached to fill out a survey did so.

\section{Study Participant Baseline Characteristics}

The majority of students (85\%) were in their didactic curriculum and had no previous clinical experience. Nine students $(15 \%)$ were in the clinical phase of their education but had experienced simulation in their didactic education within a few months to weeks of the student-run free clinic. Of the students in the clinical phase of their education, three were PA students in the second half of their second year of education, six were pharmacy students in their fourth year of their education, and one medical student was participating in clerkships (minimal clinical experiences
Table 3 Pearson correlation of development of the clinical learning environment comparison survey scales

\begin{tabular}{|c|c|c|c|c|c|c|}
\hline Scale & 1. & 2. & 3. & 4. & 5. & 6. \\
\hline 1. Communication & & $0.49 * *$ & $0.56 * *$ & $0.51 * *$ & $0.35 * *$ & $0.58 * *$ \\
\hline 2. Healthcare process & & & $0.54 * *$ & $0.68 * *$ & $0.71 * *$ & $0.62 * *$ \\
\hline 3. Holism & & & & $0.60 * *$ & $0.52 * *$ & $0.46 * *$ \\
\hline 4. Critical thinking & & & & & $0.59 * *$ & $0.48 * *$ \\
\hline 5. Self-efficacy & & & & & & $0.58 * *$ \\
\hline 6. Teaching-learning dyad & & & & & & \\
\hline
\end{tabular}

$* * p<.01$ 
Fig. 1 Visual representation of MANOVA analytical model including student demographic and educational factors

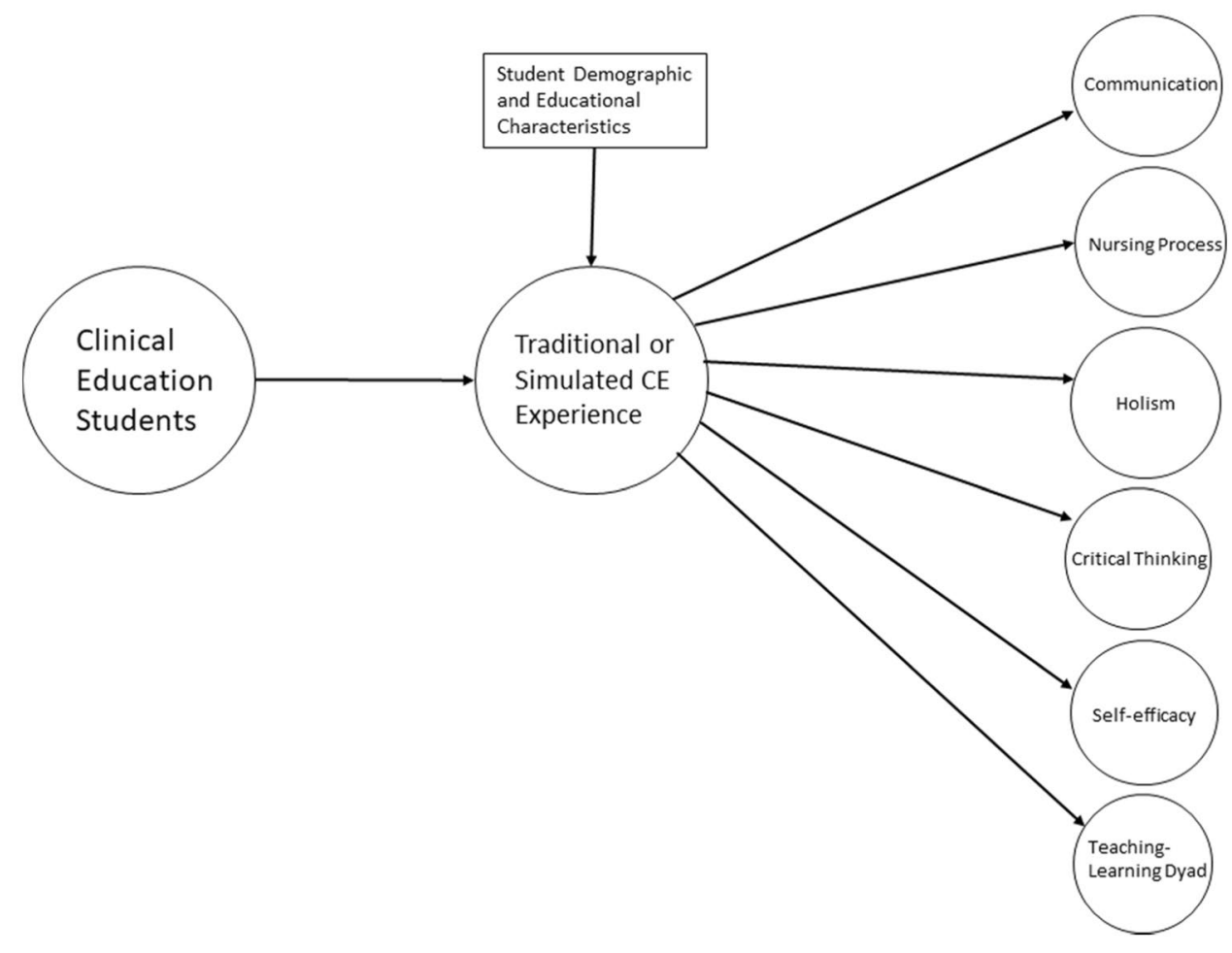

on a regular basis during the didactic phase of the curriculum) during their third year of medical school. There were a significantly higher number of pharmacy students in the clinical phase of the curriculum $(p<.001)$ when compared to physician assistant or medical students. No other significant demographic or educational differences between students were identified.

\section{CLECS Scales}

As predicted, the Pearson bivariate correlation revealed a positive and moderate to large relationship between the CLECS scales. Because all the scales were positively correlated, a multivariate analysis approach was more appropriate to minimize the type I error rate which is increased when conducting a series of univariate statistical tests [17, 18]. The overall differences in the CLECS scales collectively between traditional and previous simulated clinical experiences, the comparison and intervention groups, were assessed first using a MANOVA test (see Fig. 1). Student educational and professional factors were added to subsequent MANOVA models to determine if there were differences in CLECS scores between students of different sex, students in different programs and universities, and students with previous IPE and clinical experiences.

\section{Comparison of CLECS Subscale Scores Between Traditional and Prior Simulated Clinical Experiences}

The overall MANOVA comparing the student-run traditional and previous simulated clinical experiences was significant $(F(6,93)=4.82, p<.001$, partial $\eta 2=0.24)$. This indicated a difference in students' CLECS scale scores taken together between the two experiences. An examination of univariate ANOVA post hoc tests for each scale showed that communication $(F(1,98)=23.40, p<.001$, partial $\eta 2=.19)$, holism $(F(1,98)=9.65, p<.01$, partial $\eta 2=0.09)$, and teachinglearning dyad $(F(1,98)=5.14, p<.05$, partial $\eta 2=0.05)$ scores were all significantly higher for the student-run traditional clinical experience compared to previous simulated experience (see Fig. 2).

\section{CLECS Scale: Student Demographic and Educational Characteristics}

Additional student demographic and educational variables were included with the type of clinical experience in separate models. The main effects and interaction of these variables with clinical experience were assessed. University attended, previous IPE experience, or whether students were in their clinical education were not significant predictors of CLECS scale scores in MANOVA models including clinical 

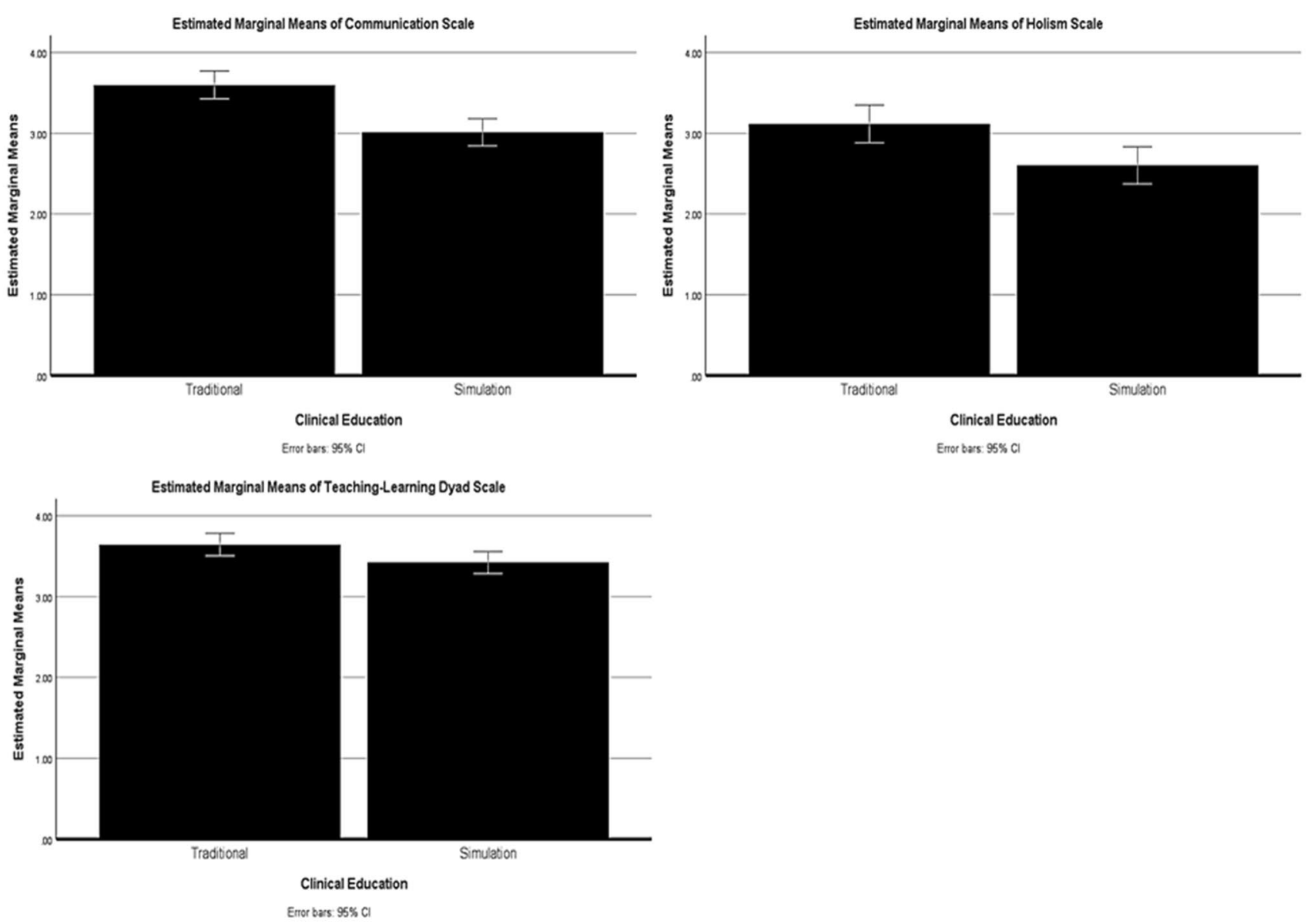

Fig. 2 Estimated marginal means of traditional and simulation clinical education for significant $(p<.05)$ individual scale ANOVAs

education type. Significant differences between other student demographic and educational groups, including student sex and program type, are discussed in further detail below.

\section{Differences Between Male and Female Students}

The interaction of student sex with clinical experience was near significant with a moderate effect size $(F(6,91)=1.81$, $p<.10$, partial $\eta 2=0.11$ ). ANOVAs showed that male students had higher simulated clinical experience scores in the teaching-learning dyad scale compared to traditional clinical experience. In contrast, female students had higher traditional clinical experience scores for teaching-learning dyad compared to simulated clinical experience $(F(1,96)=5.86$, $p<.05$, partial $\eta 2=0.06$ ) (see Fig. 3).

\section{Differences Between Physician Assistant, Pharmacy, and} Medical Students The interaction main effect of student type with clinical experience was significant $(F(12,176)=1.91$, $p<.05$, partial $\eta 2=0.12$ ). An examination of univariate
ANOVA post hoc tests showed a near significant difference for the interaction of student type and clinical education type in the communication $(F(2,92)=2.37, p<.10$, partial $\eta 2=0.05)$ and in the critical thinking $(F(2,92)=2.32$, $p<.10$, partial $\eta 2=0.05$ ) scales. Physician assistant, pharmacy, and medical students all had lower communication scale scores for prior simulated clinical experience compared to the traditional clinical experience; however, differences in physician assistant students' scores in this scale between clinical experience types were much smaller compared to pharmacy and medical students. Physician assistant and medical students had lower critical thinking scale scores for prior simulated clinical experience compared to the traditional clinical experience. In contrast, pharmacy students had higher critical thinking scale scores for prior simulated clinical experience compared to the traditional clinical experience (see Fig. 4).

\section{Qualitative Analysis}

Student surveys yielded 21 complete responses (students that answered both questions), 24 partial responses (students 
Fig. 3 Estimated marginal means of traditional and simulation clinical education between female (black) and male (gray) students. The overall MANOVA was near significant $(p<.10)$, and the individual scale ANOVA was significant at $p<.05$. Error bars: standard error of the estimated marginal means
Differences in CLECS Teaching-Learning Dyad Scale Between

Female and Male Students Participating in Traditional and Clinical Experiences

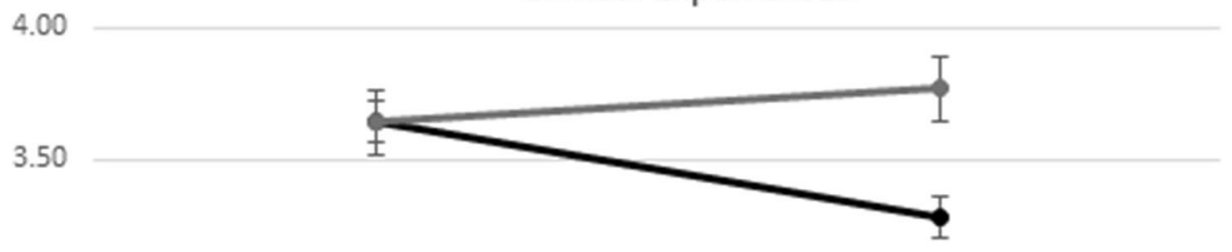

3.00

2.50

-FemaleStudents MaleStudents

2.00

1.50

1.00
Simulation that answered only one of the questions), and 15 students that did not respond to the qualitative questions. To assist in identifying the salience and saturation of themes, the researchers generated word clouds that brought the most common terms and associations to the open-ended questions. Each open-response item generated a distinct word cloud that indicated two meta-themes. The first meta-theme is that the clinical experiences develop student clinicians who are patient- and care-focused. The second meta-theme

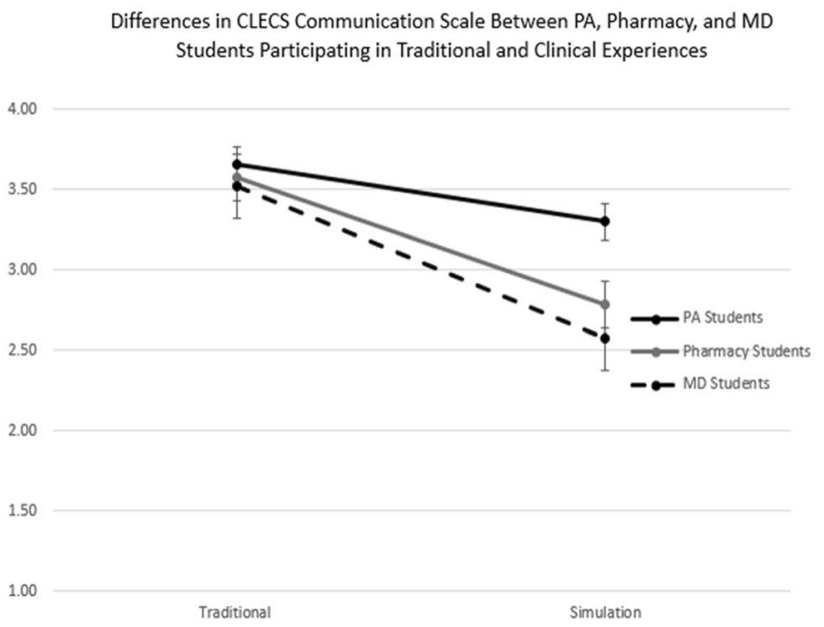

Fig. 4 Estimated marginal means of traditional and simulation clinical education between PA (black), pharmacy (gray), and MD (dashed) students. The overall MANOVA was significant $(p<.05)$, and the is related to field-specific professional development. These themes can be viewed as the external, patient-focused, and internal or professional-focused aspects of the clinical training experience.

The first meta-theme represented in Fig. 5a word cloud emphasized aspects of patient care skills. The students expressed how they could build patient care skills, such as critical thinking, communication, taking a medication history, and motivational interviewing. The word cloud

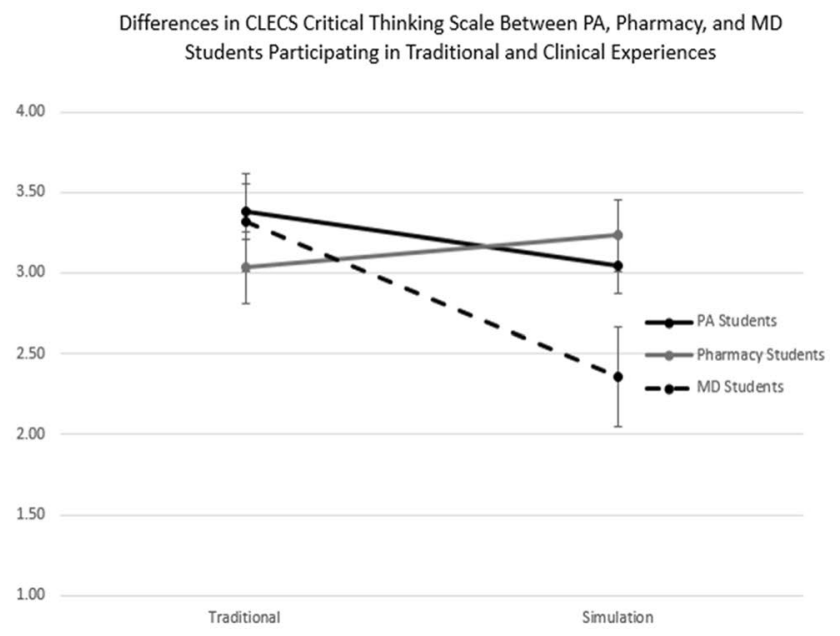

individual scale ANOVAs was near significant at $p<.10$. Error bars: standard error of the estimated marginal means 
Fig. 5 Word Clouds of Qualitative Student Survey. a Word Cloud that Emerged from the Item, "Please share specific examples how the clinical experience prepared you as a clinician." b Word Cloud that Emerged from the Item, "Please describe your interprofessional experience today"

a. Please share specific examples how the clinical experience prepared you as a clinician.

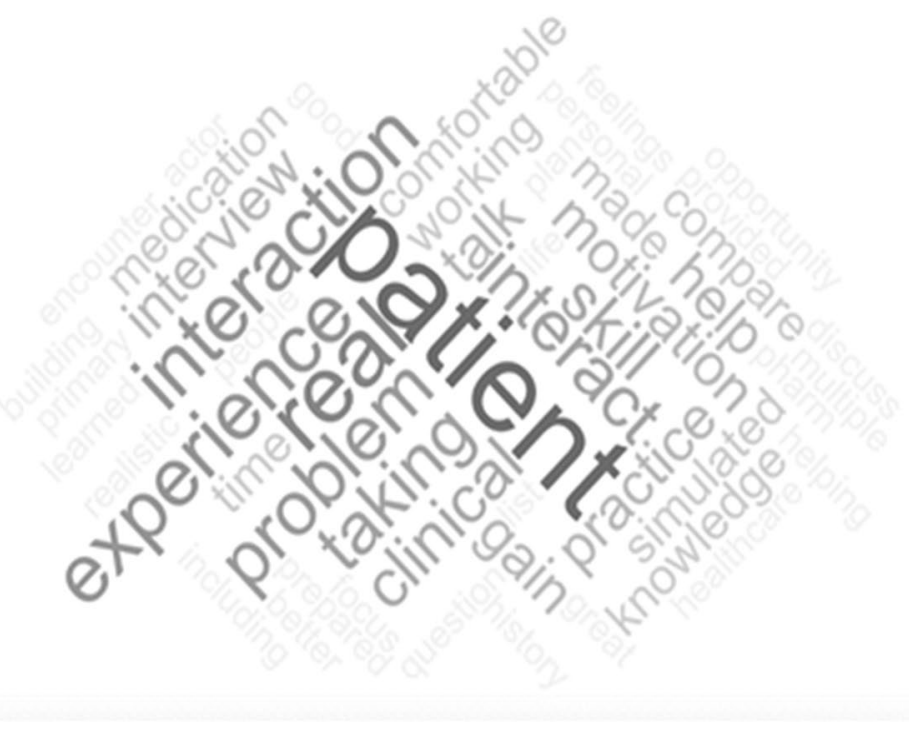

b. Please describe your interprofessional experience today.

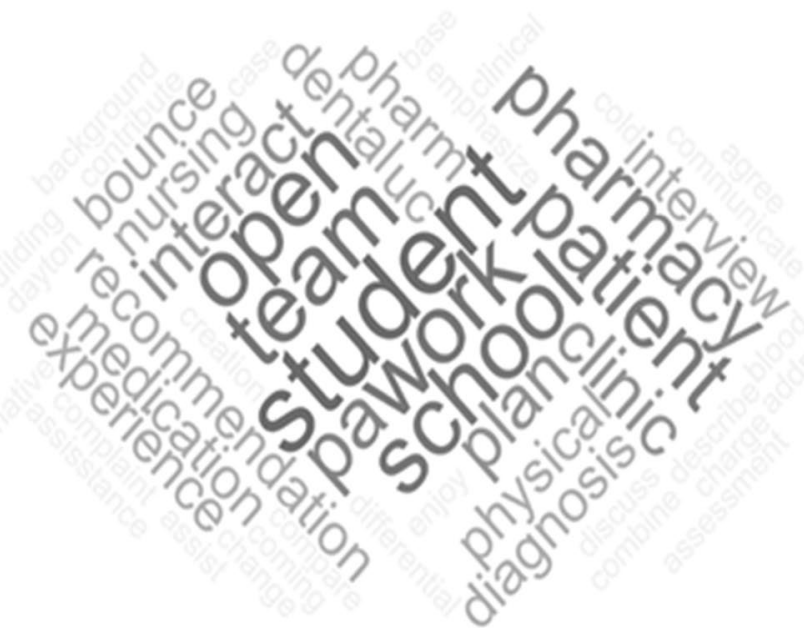

in Fig. 5a illustrates patient care, with the word patient highlighted in bold followed by words supporting clinical skills. In one of the responses, a student stated, "Direct patient interaction has helped me gain personal information, and the ability to use clinical knowledge helped a lot." In another response, a student added that the experience "helped to improve my patient communication skills, including active listening and motivational interviewing." The first meta-theme also highlighted the student's perception of increased confidence in patient-to-student clinical interaction. One student noted, "[The clinical experience helped prepare me as a clinician by] dealing with real people and their problems; dealing with their different personalities. Everything is more personal when the patient isn't an actor."

The second meta-theme denoted in the Fig. 5b word cloud underscored interpersonal collaboration, with students mentioning how they were able to interact with various members of the healthcare team. The common words that emphasized collaboration included team, student, interact, and plan. One student commented, "While going over the medication list, we were able to bounce questions off of each other. If I had trouble describing something, the physician assistant student was able to step in and assist." While another commented, "I enjoyed seeing things from a pharmacist's perspective" and "...comparing, learning, and noting other's strengths on the team". 


\section{Discussion}

This pilot study engages three health professions in an interprofessional setting and assesses student learning needs being met comparing the student-run traditional clinical compared to didactic simulation educational experience. The current study is unique due to the varied clinic topics, inclusion of pharmacy, physician assistant, and medical students as well as the assessment of the student learning needs met by the clinic in addition to the interprofessional experience. Similar student-run free clinic studies with these three professions have a specific clinic topic and either focus only on interprofessional student perceptions and/or clinical outcomes for the patients involved in the clinic.

The CLECS student assessment tool used in this study was designed to compare student learning needs being met by these two healthcare educational methods. Originally, this survey was utilized to evaluate the student's learning environment experiences for undergraduate nursing students. Nursing students believed in four of the six subscales that their needs were better met in the traditional clinical environment versus the simulated clinical environment. In the remaining two subscales, the results were equivocal. It was developed from topics identified in practice and in the simulation and nursing literature [12]. If student learning needs and positive interprofessional experiences are being identified early in a healthcare curriculum through volunteering at a student-run underserved health clinic, the sequence of clinical exposure compared to simulation experience may be considered with respect to curricular experiential learning. The use of interprofessional student-run clinics could potentially be considered as well for clinical rotations in the wake of reduced availability of clinical sites during a pandemic situation [16].

Student-run free clinics offer students the opportunity to take initiative in a clinical environment while being supervised by faculty members of a healthcare professional school. Each member of the healthcare team had the opportunity to apply their developing expertise while participating in the student-run free clinic due to the intentional pairing by precepting faculty of the pharmacy, medical, or physician assistant professions with other healthcare disciplines while caring for underserved patients in the surrounding community. Students were openly discussing various aspects of each health discipline's focus while giving input to the overall assessment and priority list of care from either the therapeutic, diagnostic, or health maintenance perspectives. More experienced team members with either therapeutics, diagnostics, or history taking offered support to the various team members with less experience in those areas. The significance of the free clinic setting also encourages students to work in collaboration with other health professionals while giving of their learned skills to help more vulnerable patients in need. Even though both education methods promote social constructivism, the free clinic setting contrasts with an OSCE or high-fidelity simulation due to the unscripted nature of the clinic setting [21,22]. Teams of learners from various health professions were tasked with navigating medically complicated patients who may have been uninsured or underinsured. Through the social interaction and collaboration with the real-life patients and healthcare team, the students were able to learn through this social and environmental setting $[21,23]$.

Students participating in the interprofessional student-run free clinics commented on the improvement of their patient care skills, medical process, patient and interprofessional communication, and critical thinking skills. The MANOVA comparing the two learning environments (traditional vs. previous simulated) indicated that students' perceptions between the two were different. Overall, student surveys demonstrated that learning needs were better met after the traditional experience in a student-run free clinic setting $(p<.001)$. There was a near statistically significant difference between male and female identified for prior simulation vs. traditional environment $(F=1.81, p<.10)$, and another analysis was significant for males preferring prior simulation experience in the learning-teaching dyad subscale $(F=5.86$, $p<.05)$. The student skills scoring higher in the traditional clinic setting included communication $(p<.001)$, holism $(p<.01)$, and the teaching-learning dyad $(p<.05)$.

Communication in the simulated setting can be limited by various factors. A simulated patient may or may not be able to include certain things in the dialogue, such as a more detailed medical history or family history that could be pertinent in the student's assessment. The actor may also have more advanced general or health literacy skills than patients in the traditional environment, who are more likely in a lower socioeconomic status or might have limited education.

Holism also scored higher in the traditional environment when compared to the simulated environment. Looking at the patient's entire medical history could play a large role in this difference. A simulated patient only has what information was given to them in preparation for the simulation. The more information a healthcare professional has about their patient, whether that be medical, social, economic, or mental health history, the more effective they can be when choosing the most appropriate and customized treatment or intervention for their chief complaint.

Students' perceptions of their learning needs for the teaching-learning dyad overall were also higher in the traditional environment. Based on survey results, students working together in groups during the traditional environment were perceived as more beneficial than when compared to 
the simulated environment. Simulated patient environments are typically designed to assess one student's clinical skill set at a time, whereas the student-run clinics are meant to include multiple students from various healthcare disciplines. A simulated environment may have very limited interaction with faculty members, if any at all. Typically, the student is not permitted to discuss the simulated experience with faculty members or other students until grading is finalized for the cohort of students. In the traditional learning environment, there are frequent interactions with faculty, which provide opportunities for immediate feedback regarding patient care and evidence-based medicine. Students are encouraged and often required to address patient concerns with a faculty member prior to adjusting a patient's care plan.

All student types had lower communication scores for prior simulated experience, suggesting that communication is learned better via traditional learning environments. Physician assistant students' scores of communication in simulation vs. traditional environments were less variable when compared to medical and pharmacy students, meaning the latter students thought the difference in communication between the two environments was greater.

Surprisingly, pharmacy students were the only healthcare discipline type that had significantly higher critical thinking scores post-simulated experience vs. traditional experience $(p<.05)$. The tendency of rating critical thinking scores higher in prior simulated experiences could also be potentially related to the higher percentage of males in the pharmacy student discipline compared to the physician assistant students ( $25 \%$ vs. $35.3 \%$ ), but this was still less than the male medical student percentage $(41.7 \%)$.

Timing variations of faculty feedback and interaction during student simulation experiences among the healthcare programs may account for these critical thinking score differences. Faculty feedback is given to the physician assistant students immediately for about a third of the simulations and within a day or two of the assessment for the remainder of simulations. Physician assistant students are assessed with simulation experience rubrics and SOAP (Subjective, Objective, Assessment, and Plan) or focused H\&P medical notes for each simulation. Pharmacy students in this study do not get immediate feedback after a simulation but are given feedback within a week after completion. Only those pharmacy students that do not pass the OSCE or meet the standards for the professional year they are in will review their performance through self-reflection after viewing the performance recording and with a faculty member. Regarding medical student simulation experiences, students receive verbal and written feedback after completion of an SPE. Medical students do not receive live feedback upon OSCE completion but do receive written feedback. Self-reflections in this setting are only required during their first professional year. Self-reflection and evaluation formats utilized by various programs aim to help students identify their strengths, weaknesses, and come up with a plan on how to improve their performances for their careers or future OSCE performances. Further research needs to be done in larger numbers to determine the reason for the simulation effectiveness differences among health profession training or if this finding is reproducible.

There was no significant difference determined between prior simulated and traditional environments when comparing the university attended, previous interprofessional experience, or if the student was in the didactic vs. clinical phase of their curriculum. This suggests that both learning environment types are perceived roughly equal as determined by the factors listed previously.

The qualitative analysis indicated that students' perceptions of the patients and their interactions with them were significant. Many students mentioned in the survey that they enjoyed patient interaction, including learning how to work with differing personalities, understanding what is important to the patient, and learning how to handle patients with multiple medical or medication issues. During a simulated clinical experience, the patient interaction may be limited. Patients in this setting are generally paid actors or unpaid volunteers that are given a script of the situation, including setting, primary problem, and potential medication issues [16]. This could potentially cause issues for a student trying to gather a large medical history on a patient, as responses in a traditional environment could vary significantly from responses given by an actor or volunteer. One student in the survey wrote, "It was very rewarding/encouraging to talk with real patients (not a simulation or patient actor)." Another student left a comment saying, "[The clinical experience helped prepare me as a clinician by] patient interaction/relationship buildinglearning to take in external factors to gain a better 'whole' picture view of [the] patient and living situation [24]."

Limitations of the study include a smaller population of students from a variety of backgrounds, the reflective nature of the simulated experience and assessing the content validity of a simulation survey for varying health professions.

This study has a smaller number of student participants due to the challenge of scheduling volunteer events to include a few different universities with demanding student didactic and clinical curriculum demands in addition to being cut short by the pandemic. Twenty-one open survey responses were also a limitation. The saturation of themes may not have been reached, but it is possible if response themes are not overly complex. Also, students were not graded for the free clinic participation so this learning experience would be considered "low stakes". The absence of a graded activity or academic consequence could have possibly influenced the perception or preference for the traditional learning environment.

The student's retrospective reflection of simulated experience within their didactic program while taking the survey is a disadvantage due to the possibility of some students having to reflect on a simulation experience that may have occurred weeks to months ago. All students except for the smaller representation of clinical students (only 15\% of participants) would 
have experienced a simulation activity in their didactic curriculum within several weeks of the free clinic. The vast majority of students were able to reflect on the didactic simulation experience compared to the free clinic experience due to not being in the clinical phase of their respective curriculum.

It is challenging to attempt matching students' didactic and interprofessional experience across disciplines, but a minority of students had already experienced clinical rotations with a higher percentage being pharmacy students. All other baseline characteristics differences were not statistically different. The vast majority of the students were preclinical, and the CLECS survey was originally designed for and validated in undergraduate nursing students [12]. Physician assistant students were the only discipline with admission requirements of at least $250 \mathrm{~h}$ of healthcare-related experience either shadowing, observing, or actively taking part in patient care. There is variation among healthcare programs regarding the format of simulations including amount and timing of faculty feedback that could have affected survey results. Clinics were similar in structure, but the supervisory experience of health care program faculty could have also varied. There was a lack of a validated survey assessing student perceptions of clinical and prior simulated experiences in various health professions in the current literature. This study received input for content validity for the three health professional student types by experts in educating these various disciplines.

Another limitation of this study is the precepting faculty volunteer time and supply investment needed to facilitate the underserved health clinics. Time constraints of university faculty make it difficult to have consistent volunteers for the underserved health clinics. Some clinics were cancelled due to unforeseen faculty academic or clinical demands. Other clinics were also cancelled due to the onset of the COVID-19 pandemic and social distancing requirements in the geographic area. Due to university budget constraints during the pandemic, supply budgets for underserved clinics were also limited.

Further study would be warranted to explore the variation in male vs. female preferences for experiential learning and healthcare profession training differences in larger numbers of study participants that may affect student perceptions of clinical experiences. Also, the potential for research regarding student-run clinics utilization in small groups or even virtually could be explored for health care education in pandemic, public health crises, or limited clinical rotation site availability.

\section{Conclusion}

In conclusion, when comparing an interprofessional student-run clinical experience vs. prior simulated learning clinical environment, students perceive their learning needs are greater met for communication, holism, and the teaching-learning dyad in the traditional learning environment for the physician assistant, pharmacy, and medical students surveyed after involvement in a volunteer health clinic. Compared to the student-run traditional clinical environment, pharmacy students felt their critical thinking skills were more improved after the simulated environment, whereas medical and physician assistant students felt that their critical thinking skills were less improved following the simulated environment. There was also a tendency toward male student preference for the simulated education experience compared to the traditional clinical experience, which could also have affected the pharmacy student's critical thinking skills improvement in the simulated environment due to a higher percentage of male pharmacy students at least compared to physician assistant students. Pharmacy, medical, and physician assistant students' communication scores were less impacted in prior simulated experience compared to the student-run traditional clinical environment. Qualitatively speaking, students perceived the interprofessional student-run clinic environment as more effective for patient communication, working with other members of the healthcare team, and learning clinical skills. Implications of this pilot study could influence the sequence of interprofessional experiential learning opportunities early in the curriculum or assist with scarce clinical rotation availability.

Acknowledgements The authors would like to thank The University of Dayton Office of Experiential Learning for Experiential Learning Innovation Faculty Fund to supply needed materials to run the underserved health clinics.

Author Contributions Amy Christopher: Research project concept, research methodology, manuscript writing, manuscript editing. Tina Gortemiller: Manuscript writing, manuscript editing, and qualitative data analysis. Jonathan Zemmer: Qualitative research, qualitative data analysis, creation of qualitative data word clouds, and writing qualitative research section of manuscript. Meredith Wronowski: Quantitative research, quantitative data analysis, quantitative data tables and figures, and writing qualitative research section of manuscript.

Funding This research did not receive any specific grant from funding agencies in the public, commercial, or not-for-profit sectors with the exception of a small grant for supplies to run health clinics. Just under one thousand dollars of the supplies used during the student run clinics were funded by a small grant entitled Experiential Learning Innovation Faculty Fund from the University of Dayton.

Availability of Data and Material The datasets generated during and/or analyzed during the current study are available from the corresponding author on reasonable request.

Code Availability Statistical analysis was performed utilizing SPSS software [17]. IBM SPSS Statistics for Windows, Version 26.0. Armonk, NY: IBM Corp.). 


\section{Declarations}

Ethics Approval This research was approved by the Institutional Review Board at the University of Dayton and Cedarville University. University of Cincinnati and Wright State University IRBs stated that student participation in the student-run free clinics and deidentified research survey use did not require a separate IRB from the original IRB obtained from the University of Dayton. Informed consent was obtained from participants by voluntary participation after being presented with a written IRB form describing the survey research.

Consent to Participate and Potential for Publication Invitation to Participate in Research Surveys and Interviews. Research Project Title: Interprofessional Healthcare Student Perceptions of Clinical Experience Effectiveness After Participation in Local Underserved Health Clinics. You have been asked to participate in a research project conducted by Amy Christopher from the University of Dayton, in the Department of Physician Assistant Program. The purpose of the project is: to determine student perceptions and preferences for traditional clinical experience compared to simulated clinical experiences in an interprofessional, underserved health clinic setting. You should read the information below and ask questions about anything you do not understand, before deciding whether to participate.

- Your participation in this research is voluntary. You have the right not to answer any question and to stop participating at any time for any reason. Answering the questions will take about $10 \mathrm{~min}$.

- You will not be compensated for your participation.

- All of the information you tell us will be confidential.

- If this is a written or online survey, only the researcher will have access to your responses. This research may result in publication of the results. If you are participating in an online survey: we will not collect identifying information, but we cannot guarantee the security of the computer you use or the security of data transfer between that computer and our data collection point. We urge you to consider this carefully when responding to these questions.

- I understand that I am ONLY eligible to participate if I am over the age of 18 .

Please contact the following investigators with any questions or concerns: Amy Christopher, achristoper1@udayton.edu, (937)-2294833. If you feel you have been treated unfairly, or you have questions regarding your rights as a research participant, you may contact Candise Powell, J.D., Chair of the Institutional Review Board at the University of Dayton, IRB@udayton.edu; Phone: (937) 229-3515.

Competing Interests The authors declare no competing interests.

\section{References}

1. Zayyan M. Objective structured clinical examination: the assessment of choice. Oman Med J. 2011;26(4):219-222. Retrieved November 15, 2019, from https://www.ncbi.nlm.nih.gov/pmc/ articles/PMC3191703/

2. Isaza-Restrepo A, Gómez MT, Cifuentes G, Argüello A. The virtual patient as a learning tool: a mixed quantitative qualitative study. BMC Med Educ. 2018;18(1):297. https://doi.org/10.1186/ s12909-018-1395-8

3. Student Run Free Clinics. (n.d.). Retrieved November 15, 2019, from https://www.studentrunfreeclinics.org/

4. Crimson Care Collaborative. (n.d.). Our Organization. Retrieved November 7, 2019, from https://crimsoncare.org/our-organization/

5. Simpson SA, Long JA. Medical student-run health clinics: important contributors to patient care and medical education. J Gen Intern Med. 2007;22(3):352-356. https://doi.org/10.1007/s11606-006-0073-4
6. Shrader S, Thompson A, Gonsalves W. Assessing student attitudes as a result of participating in an interprofessional healthcare elective associated with a student-run free clinic. J Res Interprof Pract Educ. 2010;1(3):23.

7. Tsu L, Buckley K, Early N, Jackowski R. Evaluation of multidisciplinary and pharmacy-only student-run clinics on student's perceptions of interprofessional roles. Curr Pharm Teach Learn. 2018;10(6):785-94. https://doi.org/10.1016/j.cptl.2018.03.018.

8. Reumerman MO, Richir MC, Domela Nieuwenhuis PM, et al. The clinical and educational outcomes of an inter-professional studentled medication review team, a pilot study. Eur J Clin Pharmacol. 2021;77(1):117-23. https://doi.org/10.1007/s00228-020-02972-3.

9. Seif G, Coker-Bolt P, Kraft S, Gonsalves W, Simpson K, Johnson E. The development of clinical reasoning and interprofessional behaviors: service-learning at a student-run free clinic. J Interprof Care. 2014;28(6):559-64. https://doi.org/10.3109/13561820.2014.921899.

10. Harder N. Replace is Not a Four Letter Word. Clin Simul Nurs. 2015;11(10):435-436. https://doi.org/10.1016/j.ecns.2015.07.001

11. Lie DA, Forest CP, Walsh A, Banzali Y, Lohenry K. What and how do students learn in an interprofessional student-run clinic? An educational framework for team-based care. Med Educ Online. 2016;21:31900. https://doi.org/10.3402/meo.v21.31900

12. Leighton K. Clinical: Development of the clinical learning environment comparison survey. Simul Nurs. 205;11(1), 44-51. https://doi.org/10.1016/j.ecns.2014.11.002

13. Core competencies for interprofessional collaborative practice. 2016. Retrieved November 15, 2019, from https://hsc.unm. edu/ipe/resources/ipec-2016-core-competencies.pdf

14. Gore T. The Relationship Between Levels of Fidelity in Simulation, Traditional Clinical Experiences and Objectives. Int J Nurs Educ Scholarsh. 2017;14(1). https://doi.org/10.1515/ijnes-2017-0012

15. Ladd E, Schober M. Nurse Prescribing From the Global Vantage Point: The Intersection Between Role and Policy. Policy Polit Nurs Pract. 2018. https://doi.org/10.1177/1527154418797726

16. Rolak S, Keefe AM, Davidson EL, Aryal P, Parajuli S. Impacts and challenges of United States medical students during the COVID-19 pandemic. World J Clin Cases. 2020;8(15):3136-41. https://doi.org/10.12998/wjcc.v8.i15.3136

17. SPSS I. Corp Ibm SPSS statistics for windows, version 26.0. Armonk, NY: IBM Corp, Released. 2018.

18. Mertler CA, Vannatta RA. Advanced and multivariate statistical methods. Los Angeles, CA: Pyrczak Publishing; 2002.

19. Tabachnick BG, Fidell LS. Using multivariate statistics. Upper Saddle River, NJ: Pearson; 2013.

20. Patton MQ. Qualitative research \& evaluation methods: integrating theory and practice. 4 th ed. Thousand Oaks, CA: Sage Publications; 2014.

21. Yin RK. Qualitative research from start to finish. Guilford publications. 2015.

22. Thomas A, Menon AJ, et al. Applications of social constructivist learning theories in knowledge translation for healthcare professionals: a scoping review. Implement Sci. 2014;9:54. https://doi. org/10.1186/1748-5908-9-54

23. Viret F, Vadot S, Morend L, Christen A, Bonvin R. Working with actors and non-actors as simulated patients: An advantage? Med Ed Publish. 2016;5(2):33. https://doi.org/10.15694/mep.2016. 000061

24. Creswell JW, Creswell JD. Research design: Qualitative, quantitative, and mixed methods approaches. Sage publications. 2017.

Publisher's Note Springer Nature remains neutral with regard to jurisdictional claims in published maps and institutional affiliations. 